ACTA ARITHMETICA

$\mathrm{XV}(1968)$

\section{A reduction of the Čebotarev density theorem to the cyclic case}

by

C. R. MacCuUner (East Lansing, Mich.)

\section{References}

[1] P. Bachmann, Grundlehren der Neueren Zahlentheorie, 1907, p. 248.

[2] Z. I. Borevich and I. R. Shafarevich, Number Theory, 1966, pp. 139-143.

[3] R. Dedekind, Gesammelte Mathematische Werlee, vol. I.

[4] L. E. Dickson, Introduction to the Theory of Numbers, 1929.

[5] P. G. Lejeune Dirichlet and R. Dedekind, Zahlentheorie, Suppl. XI, od. 4, I894, p. 187 .

[6] R. Fricke, Elliptische Fruntitionen, 2, 1922, p. 148.

[7] C. F. Gauss, Disquisitiones Arithmeticae, Artis., pp. 234-243.

[8] E. Hecke, Tortesungen über die Theorie der Algebraisohen Zahlen, 1023, pp. 213-217.

[9] D. Hilbert, Die Theorie der Algebraisohen Zahlloöper, \$76.

[I0] E. E. Kummer, Jour. für Mathematik, 35 , p. 325.

[11] R. König, Jahresbericht d. Deutschen Math.-Vereinigung, 22, 1913, pp. $239-254$.

[12] E. Landau, Vorlesungen iiber Zahtentheorie, 1927, v. 3, pp. 187-196.

[13] G. Pall, (a) Bull. Amer. Math. Soc. 54 (1948), pp. 1171-1175; (b) Trans. Amer. Math. Soc. 35 (1933), pp. 491-509.

[14] J. Sommer, Vortesungen ïber Zahlentheorie, 1907, pp. 197-220 (French transl. by A. Lévy, Paris, 1911, pp. 205-229).

[15] H. Weber, Math. Annalen, 48 (1897), pp. 459-462; Algebra III, 1908 , pp. $330-337$.

[16] O. Zariski and P. Samuel, Oommutative Algebra, I, pp. 246-247.

LOUISIANA STTATE UNIVERSIIYY

Baton Rouge, Louisiana

Reģu par la Rédaction le 1.12.1967
The two most useful theorems of Algebraic Number Theory are Kummer's Theorem on prime factorizations and the Čebotare $\mathrm{r}$ Density Theorem. Unfortunately until now Cebotarev's Theorem has been inaccessible to the beginning student because of its difficult proof. I present here a reduction of Čebotarev's Theorem to the case of cyclic extensions, a case that can be handled by abelian $L$-series. (See [1], page 165 and 218.)

Notation. If $k$ is a number field and $a$ a fractional ideal of $k$, then $\|\mathfrak{a}\|_{l_{c}}$ will denote the absolute norm of a (over the rational number field $\boldsymbol{Q}$ ). If $K$ is a finite galois extension of $k, p$ a prime ideal of $k$, and $\mathfrak{P}$ a prime ideal of $K$, then

$$
\left[\frac{K / k}{\mathfrak{P}}\right] \text { and }\left(\frac{\pi / k}{\mathfrak{p}}\right)
$$

will denote the Frobenius and Artin symbol respectively. If $G$ is a group, then $C_{G}(\sigma)$ and $\varkappa_{G}(\sigma)$ will denote the centralizer and the conjugacy class of $\sigma$ in $G$ respectively. Finally if $S$ is a subset of $G$, then $|S|$ will denote the cardinality of $S$.

THEOREM (Ǒebotarev Density Theorem). Let $k$ be an algebraic number field and let $K$ be a finite galois extension of $k$ with galois group $G$ over $\%$. If $\sigma$ is an element of $G$, then the Dirichlet density of all primes $\mathfrak{p}$ of 7 with

is

$$
\left(\frac{K / k}{\mathfrak{p}}\right)=x_{G}(\sigma)
$$

$$
\left|x_{G}(\sigma)\right| /(G: 1) \text {. }
$$

Reduction to the case that $K / k$ is cyclic. Let $H$ denote the cyclic subgroup of $G$ generated by $\sigma$. Suppose $\mathfrak{p}$ is a prime of $k$ with

$$
\left(\frac{K / k}{\mathfrak{p}}\right)=x_{G}(\sigma)
$$


Then ( $\mathfrak{p}$ is unramified in $K$ ) $\mathfrak{p}$ possesses a prime divisor $\mathfrak{P}$ in $K$ with

$$
\left[\frac{K / k}{\mathfrak{P}}\right]=\sigma .
$$

Let us now count the number of such prime divisors $\mathfrak{P}$ of $p$ in $K$. All such prime divisors are certainly conjugate under $G$ but what is important here is that they are conjugate under $\sigma_{G}(\sigma)$ since

$$
\left[\frac{K / k}{\tau \mathfrak{P}}\right]=\tau \sigma \tau^{-1}=\sigma
$$

implies that $\tau$ is an element of $C_{G}(\sigma)$. Therefore $p$ has exactly $\left(C_{G}(\sigma): H\right)$ prime divisors $\mathfrak{P}$ in $K$ such that

$$
\left[\frac{K / k}{\mathfrak{P}}\right]=\sigma
$$

since $H$ is the stabilizer $=$ the decomposition group of $\mathfrak{P}$.

Let $K_{Z}$ be the fixed field of $H$, i.e., the decomposition field of $\mathfrak{P}$ over $k$. Let

$$
P=\mathfrak{P} \cap K_{Z} \text {. }
$$

Then $P$ gains only degree in transit from $K_{Z}$ to $K$,

$$
\left[\frac{K / K_{Z}}{\mathfrak{P}}\right]=\sigma
$$

and

$$
\left(\frac{K / K_{Z}}{P}\right)=\varkappa_{H}(\sigma)=\{\sigma\} \text {. }
$$

Summing up, each prime p of $k$ with

$$
\left(\frac{K / k}{\mathfrak{p}}\right)=x_{G}(\sigma)
$$

has exactly $\left(C_{Q}(\sigma): H\right)$ prime divisors $P$ in $K_{Z}$ with

$$
\left(\frac{K / K_{Z}}{P}\right)=\{\sigma\}
$$

and.

$$
\|P\|_{K_{\boldsymbol{Z}}}=\|\mathfrak{p}\|_{k} \text {. }
$$

On the other hand there may be primes $P_{1}$ of $K_{Z}$ with

$$
\left(\frac{K / K_{Z}}{P_{1}}\right)=\{\sigma\}
$$

not arising in this way but these are all of degree larger than 1 over $k$. Therefore

$$
\left(C_{G}(\sigma): H\right) \sum_{\left(\frac{K / k}{\mathfrak{p}}\right)=x_{G^{(}}(\sigma)} 1 /\|\mathfrak{p}\|_{i:}^{s}=\sum_{\left(\frac{K / K_{Z}}{P}\right)=\{\sigma\}} 1 /\|P\|_{K_{Z}}^{s}+O(1) .
$$

But now let us assume that the Cebotarev Density Theorem holds for cyclic extensions and in particnlar for $K / K_{Z}$. Then we have that the Dirichlet density of all primes $p$ of $k$ with

is exactly

$$
\left(\frac{K / k}{\mathfrak{p}}\right)=\varkappa_{G}(\sigma)
$$

$$
\frac{(H: 1)}{\left(C_{G}(\sigma): 1\right)} \cdot \frac{1}{(H: 1)}=\frac{1}{\left(C_{G}(\sigma): 1\right)}=\frac{\left|\varkappa_{G}(\sigma)\right|}{(G: 1)}
$$

\section{Reference} 1967.

[1] J. W. S. Cassels, A. Froehlich ed., Algebraic Number Theory, Washington.

\section{MIOHIGAN STATE UNIVERSTTY}

East Lansing, Michigan

Reçu par la Rédaction le 2\%.12.1967 